lower adipogenic potential when cultured in specific inducing conditions.

Our results suggest that PLZF is one essential transcription factor involved in the early steps of chondrogenesis acting upstream of Sox9. Further studies will aim at determining the mechanism underlining the activation of chondrocyte-specific markers.

\title{
8 IDENTIFICATION OF PLZF AS AN OSTEOCHONDROGENIC TRANSCRIPTION FACTOR IN MESENCHYMAL STEM CELLS
}

Djouad $F_{1}^{1,2}$ Guérit $D_{1}^{1,2}$ Bony $C_{1}^{1,2}$ Jorgensen $C_{1}^{1,2,3}$ Noël $D^{1,2} \quad{ }^{1} /$ nserm U844, Montpellier, France; ${ }^{2}$ Université MONTPELLIER1, UFR de Médecine, Montpellier, France; ${ }^{3} \mathrm{CHU}$ Montpellier, Hôpital Lapeyronie, Unité Clinique d'Immuno-Rhumatologie, Montpellier, France

10.1136/annrheumdis-2011-201237.8

Mesenchymal stem cells (MSC) are suitable sources for cellbased therapies in cartilage engineering. However, their potential to regenerate a fully functional tissue relies on the presence of a differentiation factor. The identification of such a specific factor represents the major issue of this study.

Bone marrow-derived human MSC were induced to differentiate towards chondrocytes using the micropellet culture technique in presence of chondrogenic medium containing hBMP-2 for 21 days. Total RNA were hybridised on DNA microarrays (Affymetrix U133A). Quantitative RT-PCR was performed on RNA extracted at various time points during chondrogenesis. The C3H10T1/2 murine MSC line was stably transfected with a plasmid expressing PLZF and cultured in chondro-, osteo-, adipogenic conditions.

Among the 1354 differentially regulated genes during chondrogenesis, 705 genes were up regulated in MSC-derived chondrocytes. The authors first focused our attention on transcription factors and in particular, on PLZF which was shown to be significantly increased compared to undifferentiated MSC using real time PCR, as soon as day 2 of chondrogenesis. The authors thus derived stable clones of C3H10T1/2 cells over-expressing PLZF (C3-PLZF). After 21 days of culture in micropellet in presence of tumour growth factor- $\beta 3$, the authors could observe a significantly higher level of aggrecan, collagen IIB and the downregulation of collagen I in C3-PLZF compared to naïve MSC. In parallel, the authors demonstrated that PLZF over-expression increased, in a dose-dependent manner, the expression level of Sox 9 , the master chondrogenic regulator. Moreover, the engineered cells show a higher osteogenic potential than naïve C3H10T1/2 cells while displaying a 